\title{
VERBAL WREATH PRODUCTS AND GERTAIN PRODUCT VARIETIES OF GROUPS
}

\author{
R. G. BURNS \\ (Received 17 November 1965, revised 5 July 1966)
}

\section{Introduction}

Recently A. L. Šmel'kin [14] proved that a product variety ${ }^{1} \mathfrak{W} \mathfrak{B}$ is generated by a finite group if and only if $\mathfrak{U}$ is nilpotent, $\mathfrak{B}$ is abelian, and the exponents of $\mathfrak{U}$ and $\mathfrak{B}$ are coprime. Alternatively, by the theorem of Oates and Powell [13], we may say that a Cross variety is decomposable if and only if it is of the above form.

Throughout this paper such a variety will be denoted by $\mathfrak{R A}_{n}$, the class of $\mathfrak{R}$ by $c$, and the exponent of $\mathfrak{R}$ by $m: n$ is the exponent of the abelian variety $\mathfrak{A}_{n}$.

In this paper we find the least number $l=l\left(\mathfrak{R A}_{n}\right)$ such that $\mathfrak{R A}_{n}$ is generated by its $l$-generator groups but not by its $(l-1)$-generator groups. For $c=1$, that is $\mathfrak{R}$ abelian, C. H. Houghton proved, generalizing a result of Graham Higman [6], that $\mathfrak{A}_{m} \mathfrak{A}_{n}$ is generated by its 2-generator groups. In fact he showed that $C_{m}$ wr $C_{n}$ generates the product variety (unpublished). (Here $C_{m}, C_{n}$ denote cycles of orders $m, n$.) As a generalization of this it can be shown easily from the structure of the critical groups in $\mathfrak{R A}_{n}$, obtained in $\S 2$, that the verbal wreath product $W_{c}=F_{c}(\mathfrak{R}) w r_{\mathfrak{M}} F_{c}\left(\mathfrak{A}_{n}\right)$ generates $\mathfrak{R A}_{n}$, where $F_{0}(\mathfrak{N})$ and $F_{c}\left(\mathfrak{U}_{n}\right)$ are the free groups of rank $c$ of $\mathfrak{R}$ and $\mathfrak{A}_{n}$ respectively. This yields $l \leqq 2 c$ immediately. However the following theorem is proved.

THEOREM. The variety $\mathfrak{R A}_{n}$, where $\mathfrak{R}$ is any nilpotent variety of class $c>1$ and exponent $m$, with $(m, n)=1$, is generated by its c-generator groups but not by its $(c-1)$-generator groups. That is $l\left(\mathfrak{N A}_{n}\right)=c$.

The precision of this result contrasts with the result of Higman [6], that any nilpotent variety of class $c$ is generated by its $c$-generator groups, and the result of Gilbert Baumslag, B. H. Neumann, Hanna Neumann and Peter M. Neumann [1], that even for the variety $\mathfrak{B}$ say, of all nilpotent groups of class $\leqq c$, we have

$$
[c / 2] \leqq l(\mathfrak{B}) \leqq c,
$$

1 This and other terms used here seem to be standard now. Full definitions may be found in $[6]$ and $[13]$. 
with no more precise bounds yet known.

In $\S 2, l\left(\mathfrak{R Q}_{n}\right) \leqq c$ is proved (this is due to L. G. Kovács) and in $\S 5$ the properties of $W_{c}$ are exploited to give the reverse inequality.

It is also shown in $\S 2$ that every critical group in $\mathfrak{R A}_{n}$ needs at most $c+1$ generators and in $\S 3$ critical groups actually requiring $c+1$ generators are constructed for $c=2$ and certain pairs of exponents $m, n$. (Cf. the result of Paul M. Weichsel [15] that critical groups of class $\leqq c$ are $c$-generator.)

This, together with the result of Hanna Neumann (Theorem 3.1) that a variety generated by a single critical group which is strictly $k$-generator, cannot be generated by its $(k-1)$-generator groups, prompts the following question. Can one construct, for any pair of integers $k, l>0$, a variety generated by its $k$-generator groups and also by a set $S$ of critical groups some of which are strictly $(k+l)$-generator? This question is answered affirmatively for $k=2$ and arbitrary $l$, mainly by reference to [2]. However, if we insist that in the above question not all of the critical groups in $S$ requiring $>k$ generators be redundant; that is, there exist $G \in S$ requiring $>k$ generators such that $\operatorname{var}(S) \neq \operatorname{var}(S \backslash\{G\})$, then the question remains completely unanswered.

In $\S 4$ a characterization of the normal closure of the top group of an arbitrary verbal wreath product is obtained. This generalizes a corresponding result of Peter M. Neumann [12] on the standard restricted wreath product.

In $\S 5$, a special case of the main result of $\S 4$ (Corollary 4.2) and an embedding theorem for a more restricted class of verbal wreath products, are used to prove $\left.l(\mathfrak{A Q})_{n}\right) \geqq c$. The embedding theorem is also contained in A. L. Smel'kin's paper [14] but the proof given here is short and more direct.

We shall denote the least number of generators required to generate a group $G$ by $d(G)$. The definition and some properties of the verbal wreath product may be found in [14] from which also the notation has been taken over.

I am grateful to Professor Hanna Neumann and Dr. L. G. Kovács for their great help and encouragement. In particular I thank Professor Neumann for many corrections and for her help in writing this paper. I also thank the referee for his careful criticism and for several suggestions which have been incorporated into the paper.

This work was done under an Australian National University research scholarship at Canberra.

\section{Upper bounds}

In this section we prove that:

$2.1 d(G) \leqq c+1$ for any critical group $G$ in $\mathfrak{R A}_{n}$ and for all $c \geqq 1$;

2.2. $l\left(\mathfrak{R A}_{n}\right) \leqq c$ for all $c>1$. 
Proof of 2.1. Firstly, for all $c \geqq 1$ we obtain the bound $2 c$ for $d(G)$ where $G$ is any critical group in $\mathfrak{R A}_{n}$. The following argument is due to L. G. Kovács and is derived in part from an argument in the paper [7] of Kovács and Newman.

Lemma 2.4.2 of Oates and Powell [13] is used:

2.3. If a group $G$ has a set of normal subgroups $M_{1}, \cdots, M$, and a subgroup $L$ such that

2.3.1 $G=L M_{1} \cdots M_{s}$;

2.3.2 $G$ is not generated by $L$ together with any proper subset of the set $\left\{M_{1}, \cdots, M_{s}\right\}$;

2.3.3 $\left[M_{\pi(1)}, \cdots, M_{\pi(s)}\right]=E$, the unit subgroup, for every permutation $\pi$ of the integers $1, \cdots, s$;

then $G$ is not critical.

Now return to the critical group $G$ in $\mathfrak{R A}_{n}$. We may assume $G$ is nonabelian since otherwise $G$ is cyclic and $d(G)=1$. By definition, a critical group is finite. Let $F=F(G)$ be the greatest normal nilpotent subgroup (the Fitting subgroup) and $\Phi=\Phi(G)$ the Frattini subgroup of $G$. Since $\Phi$ is nilpotent and normal, $\Phi \leqq F . F$ is a $p$-group for some prime $p$, otherwise $G$ would not be monolithic (i.e. it would not possess a unique minimal normal subgroup). Furthermore $p \mid m$ where $m$ is the exponent of $\mathfrak{R}$. For, by the definition of a product variety and since $G$ has been assumed nonabelian, $G$ is the extension of a non-trivial subgroup $S$ in $\Re$ by a group in $\mathfrak{A}_{n}$, and we must have $S \leqq F$. Since $(m, n)=1$ there is, by the SchurZassenhaus Theorem, a complement $L$ of $F$ in $G$. It follows that $L \Phi / \Phi(\cong L)$ is a complement of $F / \Phi$ in $G / \Phi$. By Theorems 2,5 and 9 of Gashütz [4], $F / \Phi$ is an elementary abelian $p$-group (Clearly $\Phi \neq F$.) Write

$$
F / \Phi=M_{1} / \Phi \times \cdots \times M_{s} / \Phi
$$

where $M_{i} / \Phi, i=1, \cdots, s$, is an elementary abelian minimal normal subgroup of $G / \Phi$. That such a decomposition exists is a consequence of Maschke's Theorem (see for example [5] p. 253): $F / \Phi$ may be regarded as an $L / \Phi$ module over $G F(p)$. Then $G=L M_{1} \cdots M_{s}$ and conditions 2.3.1 and 2.3.2 are satisfied. If $s>c$, condition 2.3 .3 will also be satisfied, contrary to the criticality of $G$. Thus $s \leqq c$.

Since $\Phi$ is the (finite) set of non-generators of $G, d(G / \Phi)=d(G)$. Therefore we can restrict our attention to $G / \Phi$. Write $G / \Phi=G_{1}, L \Phi / \Phi=L_{1}$, $F / \Phi=F_{1}$, and $M_{i} / \Phi=N_{i}, i=1, \cdots, s$. Let $x_{i}$ be any non-trivial element from $N_{i}, i=1, \cdots, s$. Then

2.4

$$
G_{1}=\operatorname{sgp}\left\{L_{1}, x_{1}, \cdots, x_{s}\right\}
$$

since the conjugates of $x_{i}$ under $L_{1}$ must together generate the whole of 
the minimal normal subgroup $N_{i}$. We now bound $d\left(L_{1}\right)$. Let $K_{i}$ be the kernel of the representation of $L_{1}$ on $N_{i}, i=1, \cdots, s$ : that is $K_{i}=C_{L_{1}}\left(N_{i}\right)$ $\triangleleft L_{1}$. By Theorem 10 of $[4], F(G / \Phi)=F / \Phi$. This, together with the abelianness of $L_{1}$, implies that $C_{G_{1}}\left(F_{1}\right) \leqq F_{1}$ : that is, $L_{1}$ is faithfully represented on $F_{1}=N_{1} \times \cdots \times N_{i}$. Therefore $\bigcap_{i=1}^{z} K_{i}=E$. Now $L_{1} / K_{i}$ is abelian and is represented faithfully and irreducibly on $N_{i}$. By a classical theorem of representation theory, this implies that $L_{1} / K_{i}$ is cyclic. Thus $L_{1}$ contains $s$ normal subgroups intersecting trivially and with cyclic factor groups. It follows that $L_{1}$ is embeddable in the direct product $L_{1} / K_{1} \times \cdots \times L_{1} / K_{\text {s }}$ of $s$ cycles and that $d(L) \leqq s$. We have $d(G)=$ $d\left(G_{1}\right) \leqq 2 s \leqq 2 c$.

Secondly, we reduce this bound to $c+1$ with the help of the following lemma.

2.5 LEMMA. Let $B$ be a finite abelian group with $\leqq s$ generators and let $B_{1}, \cdots, B_{,-1}$ be $s-1$ subgroups such that $B / B_{i}$ is cyclic, $i=1, \cdots, s-1$. Then there exists a set $\left\{g_{1}, \cdots, g_{s}\right\}$ of generators of $B$ such that $g_{i} \in B_{i}$ for $i=1, \cdots, s-1$.

Proof. If $B$ is trivial then so also is the lemma. Assume $B \neq E$.

Write $B$ as the direct product of its Sylow subgroups: $B=S_{p_{1}} \times \cdots \times S_{p_{k}}$ say. Then, for each $j, \mathbf{1} \leqq j \leqq k, S_{p_{s}}$ and $B_{1} \cap S_{p_{j}}, \cdots, B_{s-1} \cap S_{p_{j}}$ satisfy the conditions of the lemma. If there exists a set $\left\{g_{i p_{j}}, \cdots, g_{s p_{j}}\right\}$ of generators of $S_{p_{i}}$ such that $g_{i_{j}} \in B_{i} \cap S_{p_{j}}, i=1, \cdots, s-1$, put

$$
g_{i}=\prod_{j=1}^{k} g_{i p_{j}}
$$$$
i=1, \cdots, s .
$$

Then $\left\{g_{1}, \cdots, g_{s}\right\}$ satisfies the requirements of the lemma.

Hence we may assume $B$ is a $p$-group. We use induction on $s$. For $s=1$ the lemma holds vacuously. Suppose $s>1$ and assume the lemma true for $s-1$. Write $B=C_{p n_{1}} \times \cdots \times C_{p n_{s}}$ where $n_{i} \geqq 0, i=1, \cdots, s$, and $n_{s} \geqq n_{j}, j<s$, and let $y_{i}$ be a generator of $C_{p^{n_{i}}}$. Consider the case $B_{1}<B$. In this case there exists some element $y=y_{1}^{\alpha_{1}} \cdots y_{s-1}^{\alpha_{s}} y_{s} \notin B_{1}$ since elements of this form generate $B$. Hence $B=C_{p^{n_{1}}} \times \cdots \times C_{p^{n_{2-1}}} \times s g p\{y\}=$ $A \times \operatorname{sg} p\{y\}$ say. For this decomposition of $B$, the projection of $B_{1}$ on $A$ must be the whole of $A$ since, if $a \neq 1$ were not in this projection then, modulo $B_{1}$, the set $\{a, y\}$ would generate a non-cyclic group. Thus for $B_{1} \leqq B$ there is a generating set $\left\{c_{1}, \cdots, c_{s-1}, g_{s}\right\}$ for $B$ such that $c_{1}, \cdots, c_{,-1} \in B_{1}$.

Write $H=\operatorname{sg} p\left\{c_{1}, \cdots, c_{s-1}\right\} \leqq B_{1}$ and consider the cyclic group $H B_{i} \mid B_{i} \cong H / H \cap B_{i}, i=2, \cdots, s-1$. The groups $H$ and $H \cap B_{2}, \cdots$, $H \cap B_{s-1}$ satisfy the conditions of the lemma and so, by the inductive hypothesis, there exist generators $g_{1}, \cdots, g_{s-1}$ for $H$ such that 
$g_{i} \in H \cap B_{i} \leqq B_{i}$, for $i=2, \cdots, s-1$. Since $g_{1} \in B_{1}$, the set $\left\{g_{1}, \cdots, g_{s}\right\}$ satisfies the requirements of the lemma. This completes the proof of the inductive step and the proof of the lemma.

For the application of this we return to 2.4: $G_{1}=\operatorname{sg} p\left\{L_{1}, x_{1}, \cdots, x_{s}\right\}$. $L_{1}$ and $K_{1}, \cdots, K_{s-1}$ satisfy the conditions of 2.5 and hence there is a generating set $\left\{l_{1}, \cdots, l_{s}\right\}$ for $L_{1}$ with $l_{i} \in K_{i}$ for $i=1, \cdots, s-1$. Since $K_{i}$ centralizes $x_{i}$ and they have coprime orders, the set $\left\{l_{1} x_{1}, \cdots, l_{s-1} x_{s-1}\right.$, $l_{s}, x_{s}$ \} generates $G_{1}$. This completes the proof of 2.1 .

One further lemma will complete the proof of 2.2. Since $\mathfrak{N A}_{n}$ is generated by its critical groups we have immediately from 2.1 that $l\left(\mathfrak{R A}_{n}\right) \leqq c+1$. In $\S 3$, for $c=2$ and certain $m, n$, critical groups in $\mathfrak{R A}_{n}$ having not fewer than 3 generators are constructed. Thus we cannot hope to obtain the upper bound $c$ for $l\left(\mathfrak{R A}_{n}\right)$ by considering the critical groups alone. However, L. G. Kovács has proved the following result.

2.6 Lemma. For each critical group $G$ in $\mathfrak{R A}_{n}$ with $s>1$ defined as previously, there exists an s-generator permutational verbal wreath product lying in $\mathfrak{R A}_{n}$ and having $G$ as a factor.

Proof. We retain the notation of the proof of 2.1 for the relevant subgroups etc. of the critical group $G$. Identify $L_{1}$ with $L$ under the mapping $l \Phi \rightarrow l, l \in L$. Then $L / K_{i}, i=1, \cdots, s$, is a cyclic group of order dividing $n$. Let $Z_{i}$ be a group isomorphic to $L \mid K_{i}, i=1, \cdots, s$, such that $Z_{j} \cap Z_{k}=\emptyset$ for $j \neq k$. Let $\theta_{i}: L / K_{i} \rightarrow Z_{i}$ be an isomorphism, $i=1, \cdots, s$. Form the set-theoretical union $\bigcup_{i=1}^{*} Z_{i},=Z$ say. Then for each $z_{i} \in Z_{i}, i=1, \cdots, s$, a permutation $\zeta_{i}$ of $Z$ is defined as follows:

$$
\begin{aligned}
& z \zeta_{i}=z z_{i} \text { if } z \in Z_{i} \\
& z \zeta_{i}=z \quad \text { if } z \notin Z_{i} .
\end{aligned}
$$

The group $Q$ generated by all such permutations is isomorphic to $Z_{1} \times \cdots \times Z_{8}$ and the restriction to $Z_{i} \subseteq Z$ of this group of permutations is the right regular representation of $Z_{i}$. Take $|Z|$ distinct isomorphic copies of the $p^{\alpha}$-cycle $C_{p \alpha}$ where $p^{\alpha}$ is the exponent of $F(G)$, and denote them by $C_{p^{x}}(z), z \in Z$. We form the verbal $\mathfrak{N}$-product (see S. Moran, [9])

$$
B=\prod_{z \in Z}^{\Re} C_{p^{x}}(z)
$$

and split-extend $B$ by $Q$ in the usual way for permutational wreath products: that is, the action of $\zeta \in Q$ on $C_{p^{a}}(z)$ is defined by

$$
(a(z))^{6}=a(z \zeta)
$$

for $a \in C_{p a}$. Then $B Q$ is the permutational verbal wreath product mentioned in the lemma. Obviously $B Q \in \mathfrak{R A}_{n}$. 
We now choose a subgroup of $B Q$ and find an epimorphism from this subgroup onto $G$. Let $\psi$ be the isomorphism $Z_{1} \times \cdots \times Z_{s} \rightarrow Q$ defined by

$$
z_{i} \psi=\zeta_{i}, \quad z_{i} \in Z_{i}, \quad i=1, \cdots, s .
$$

Then the mapping

$$
\theta: l \rightarrow\left(l K_{1}\right) \theta_{1} \psi \cdot\left(l K_{2}\right) \theta_{2} \psi \cdots\left(l K_{s}\right) \theta_{s} \psi, l \in L,
$$

is a monomorphism of $L$ into $Q$. Write $L \theta=L_{2} \leqq Q$. Let $b_{i} \in M_{i} \backslash \Phi(G)$, $e_{i}$ be the identity of $Z_{i}, i=1, \cdots, s$, and let $a$ generate $C_{p^{\alpha}}$. Then the mapping

$$
\begin{aligned}
a\left(e_{i}\right) \rightarrow b_{i}, & i=1, \cdots, s \\
l \theta \rightarrow l, & l \in L,
\end{aligned}
$$

can be extended to an epimorphism of $B L_{2}$ onto $G$. This follows from the structure of $G$ obtained in the proof of 2.1, from the freeness of $B$ in $\Re$ and the well-known von Dyck's Theorem ([8], Vol. I, p. 130).

Finally we find the number of generators sufficient to generate $B Q$. If $s=1, B Q$ is 2-generator. If $s>1, B Q$ is $s$-generator: for, if $a\left(e_{i}\right)$ generates $C_{p x}\left(e_{i}\right)$ and $z_{i}$ generates $Z_{i}, i=1, \cdots, s$, then $B Q$ is generated by the set $\left\{a\left(e_{i}\right), z_{i} \psi \mid i=1, \cdots, s\right\}$; but $a\left(e_{j}\right)$ and $z_{k} \psi$ commute if $j \neq k$ and have comprime orders and hence $\left\{a\left(e_{i}\right) \cdot\left(z_{(i+1) \bmod s} \psi\right) \mid i=1, \cdots, s\right\}$ also generates $B Q$. This completes the proof of 2.6 and hence 2.2 .

\section{Gritical groups}

Firstly we construct the critical groups promised in $\S 1$, to show that the bound $c+1$ for the least number of generators of the critical groups in $\mathfrak{N Q}_{n}$ is best possible, at least in some cases.

We know from 2.2 that, in particular, for $c=2, \mathfrak{N A}_{n}$ is generated by its 2-generator groups. It is also generated by its critical groups.

For a pair of primes $p, q$ related in a way to be described, we shall construct a critical group $G=G(p, q)$ of exponent $p q$ which is strictly 3-generator and is an extension of a nilpotent group of class 2 and exponent $p$, by an abelian group of exponent $q$.

Suppose a prime $p>2$ is given arbitrarily and let $q$ be any prime dividing $p-1$. Then there exists an $r, 1<r<p$, such that $r^{a} \equiv 1(\bmod p)$ since the non-zero integers modulo $p$ form the multiplicative group of $G F(p)$. Suppose $N$ is the reduced free group of exponent $p$ and class 2 on two free generators $a, b$, and let $x$ generate a cycle of order $q . G$ is the splitting extension of $N$ by $\operatorname{sg} p\{x\}$ obtained by defining $a^{x}=a^{r}, b^{x}=b^{r}$. Thus

$$
G=g p\left\{N, x \mid x^{a}=1, a^{x}=a^{r}, b^{x}=b^{r}\right\} .
$$


$G$ is critical because any proper factor of $N$ is abelian whence it follows easily that any proper factor of $G$ is metabelian, whereas $[a, x]=a^{r-1}$ and $[b, x]=b^{r-1}$ generate the non-abelian group $N$, so that $G$ itself is not metabelian.

For the verification of $d(G)=3$ we proceed as follows. If $G$ is 2generator, some pair $\{x u, v\}, u, v \in N$, will do to generate it. There exists $w \in N$ such that $w^{r-1}=u$ since $(r-1, p)=1$. Thus $x u=x w^{r} w^{-1}=$ $x w w^{x} w^{-1}=w x w^{-1}$ and $G=\operatorname{sg} p\left\{x, v^{\prime}\right\}$ where $v^{\prime}=w^{-1} v w$. However, modulo $\operatorname{sg} p\{[a, b]\}$, the monolith of $G, s g p\left\{x, v^{\prime}\right\}$ is an extension of $C_{p}$ by $C_{d}$, whereas $\bmod s g p\{[a, b]\}, G$ is an extension of $C_{p} \times C_{p}$ by $C_{q}$. Thus we have reached a contradiction and $d(G)=3$.

Let $\mathfrak{B}$ be a variety generated by its critical groups and also by its $k$ generator groups, for some finite $k$. We now touch briefly on the question whether or not there exists a connexion between $l(\mathfrak{B})$ and the numbers of generators required by the critical groups in $\mathfrak{B}$.

We prove firstly the following theorem.

3.1 Theorem. (Hanna Neumann) The variety generated by a single critical group $G$, with $d(G)=k$, is not generated by its $(k-1)$-generator groups.

Proof. Suppose the theorem false and that $G$ is a counterexample, $G$ critical, $d(G)=k$. Denote by $F_{k-1}$ the reduced free group of rank $k-1$ of $\operatorname{var}(G)$. Then $\operatorname{var}(G)=\operatorname{var}\left(F_{k-1}\right)$ by the supposition. Hence $F_{k-1}$ is isomorphic to a factor of a cartesian power $G^{I}$ where $I \neq \emptyset$ is some index set. Since $F_{k-1}$ is free in $\operatorname{var}(G)$, it is in fact embeddable in $G^{I}: F_{k-1} \cong A \leqq G^{I}$, say. Let $\theta_{i}, i \in I$, be the projection of $G^{I}$ on its $i$-th co-ordinate. If $A_{i} \cong G$ for some $i \in I, G$ would be isomorphic to a factor group of $A \cong F_{k-1}$ and would then have fewer than $k$ generators. Thus $A \theta_{i}$ is isomorphic to a proper subgroup of $G$ for all $i \in I$. Hence $F_{k-1}$ is in the variety generated by all $A \theta_{i}$ which is in turn in the variety generated by the proper subgroups of $G$. This gives us a contradiction and completes the proof.

This gives rise to the question asked in $\S 1$.

It can be proved (see [2], chapter 6) that if $\mathfrak{B}$ (as above) is also Cross, then it is generated by its $k$-generator critical groups. Assuming that $l\left(\mathfrak{R Q}_{n}\right)=c$ (the proof of this is completed in $\S 5$ ) it follows that the Cross variety $\mathfrak{N A}_{n}$ must contain at least one critical group $G$ with $d(G)=c$. Hence there exists for each $c \geqq 1$, a critical group $G_{o}$ such that $d\left(G_{c}\right)=c$. The group $G_{c}$ can be embedded in some finite symmetric group $S(c)$ say, which has a set of two generators. Thus $\operatorname{var}(S(c))$ is generated by its 2generator groups (and its critical groups since it is Cross) and contains at least one critical group requiring $c$ generators. This answers the question asked in $\S 1$, for $k=2$. 
However the question of the existence of a set of critical groups some of which require $>k$ generators, generating irredundantly (in the sense given in $\S 1$ ) a variety generated by its $k$-generator groups, remains unanswered.

An an example of what may happen, let us return for the moment to the variety $\mathfrak{N O}_{a}$, where $\mathfrak{N}$ is assumed to be of class 2 and exponent $p>2$, and the primes $p, q$ are such that $q \mid p-1$. (There is only one such variety $\mathfrak{N}$.) In [2], chapter 6, the complete subvariety lattice of $\mathfrak{N A}_{a}$ is determined by means of its critical groups. It turns out that the strictly 3-generator group $G=G(p, q)$ constructed above is the only such in $\mathfrak{R A}_{q}$, and that if $\mathfrak{B}$ is any subvariety of $\mathfrak{R A}_{a}$ containing the critical group $G$, and such that $l(\mathfrak{B})=2$, then $G$ can be omitted from any set $S$ of critical groups generating $\mathfrak{B}$. That is, if $\mathfrak{B}=\operatorname{var}(S)$ then $\mathfrak{B}=\operatorname{var}(S \backslash\{G\})$.

\section{The verbal wreath product}

In this section we prove a theorem on the standard verbal wreath product $W=A w r_{3} B$ where $A$ and $B$ are arbitrary non-trivial groups and $\mathfrak{B}$ is an arbitrary variety. It is a generalization of Theorem 4.1 of [12] and part of the proof is a generalization of the proof in [12].

Since our only concern from now on will be with the standard verbal wreath product, the adjective "standard" will, for brevity, be dropped.

The theorem is applied in $\S 5$ in the special case $V(A)=E$ (Corollary 4.2) to obtain a lower bound for $l\left(\mathfrak{N A}_{n}\right)$. For this special case a simpler proof is possible. However the full theorem is of independent interest and not much is saved by proving only the weaker version.

Before we can state the theorem, some definitions are needed. Suppose $F$ is a free group on a countably infinite set $\left\{x_{1}, x_{2}, \cdots\right\}$ of free generators. Let $V$ be any fully invariant subgroup of $F$ and $D$ the derived group of $F$. For any group $G$ let $V(G)$ denote the verbal subgroup of $G$ determined by $V$, and $\mathfrak{B}$ the variety of all groups $G$ for which $V(G)=E . V$ and $D$ are verbal subgroups of $F$ and therefore $V \cap D$ is fully invariant and hence also a verbal subgroup. It is easy to verify that, for any group $G$, $(V \cap D)(G) \leqq V(G) \cap D(G)$. In view of subsequent arguments it is worth while pointing out that $\mathrm{B}$. H. Neumann [11] has found an example where the inequality is strict. (Cf. also S. Moran [10].). Finally we shall use without comment that $V(G \theta)=V(G) \theta$ for every homomorphism $\theta$.

Let $K$ denote the base group of $W$. Then we have the following result.

4.1 TheOREM. Let $B_{1}>E$ be normal in $B, T_{1}$ be any transversal for $B_{1}$ in $B$, and $P$ denote the verbal product $\prod_{t \in T_{1}}^{\mathfrak{g}} A(t)<K$. Then 


$$
\begin{aligned}
B_{1}^{w} \cap K & =\left[B_{1}, K\right]= \\
& \left\{\alpha_{1}^{b_{1}} \alpha_{2}^{b_{2}} \cdots \alpha_{r}^{b_{r}} \mid r \geqq 1 ; b_{i} \in B_{1}, \alpha_{i} \in P, \text { for } i=1, \cdots, r ;\right. \\
& \left.b_{j} \neq b_{j+1} \text { for } j=1, \cdots, r-1 ; \alpha_{1} \alpha_{2} \cdots \alpha_{r} \in(V \cap D)(P)\right\} .
\end{aligned}
$$

Here $B_{1}^{* 0}$ denotes the normal closure of $B_{1}$ in $W$.

The proof consists of a string of lemmas. The first two, 4.1.1 and 4.1.2, reduce the problem to the case $B_{1}=B$. Lemma 4.1.1 is well known and the proof is omitted.

4.1.1 Lemma. If $B_{2}$ is any subgroup of $B$ and $T_{2}$ is any left transversal for $B_{2}$ in $B$, then

$$
B_{2} \cdot K \cong\left(\prod_{t \in T_{2}}^{\mathfrak{g}} A(t)\right) w_{\mathfrak{2}} B_{2} .
$$

If, in the right hand side, we interpret the action of $B_{2}$ on $\prod_{t \in T_{2}}^{\mathfrak{g}} A(t)$ as in $W$, we may (and shall in future) replace $\cong$ by $=$.

4.1.2 Lemma. The normal closure of $B_{1}$ in $W$ is $B_{1}\left[B_{1}, K\right]$.

Proof. The subgroup $\left[B_{1}, K\right]$ of $W$ is normalized by $K$ : for, if $b_{1} \in B_{1}$; $k, k_{1} \in K$, then

$$
\left[b_{1}, k\right]^{k_{1}}=\left[b_{1}, k_{1}\right]^{-1}\left[b_{1}, k k_{1}\right] \in\left[B_{1}, K\right] .
$$

Also, $\left[B_{1}, K\right]$ is normalized by $B$ since $B_{1} \unlhd B$ and $K \triangleleft W$. Hence $\left[B_{1}, K\right]$ is normal in $B K=W$.

The subgroup $B_{1}\left[B_{1}, K\right]$ is obviously normalized by $B$. Also, if $k \in K$, $b_{1} \in B_{1}$, then

$$
b_{1}^{k}=b_{1}\left[b_{1}, k\right] \in B_{1}\left[B_{1}, K\right] .
$$

Hence $B_{1}\left[B_{1}, K\right]$ is normal in $W$ and must be the normal closure of $B_{1}$ in $W$.

4.1.3 CoRollary. The normal closure of $B_{1}$ in $W$ is the same as its normal closure in $B_{1} K$.

This follows at once from Lemma 4.1.2. This corollary, together with Lemma 4.1.1, allows us to assume that $B_{1}=B$. The following few lemmas are concerned with this case and with a particular verbal wreath product.

We consider in particular the verbal wreath product $W^{*}=A w r_{*} B$ (which is in fact isomorphic to the free product $A^{*} B$ - but we shall not need this) since the more general wreath product $W$ is a factor group of it. The base group of $W^{*}$ is the free product $\Pi_{b \in B}^{*} A(b)=\Pi^{*} A(b)$, say. Now by the definition of the verbal product,

$$
K=\prod_{b \in B}^{\mathfrak{g}} A(b)=\Pi^{*} A(b) /\left(V\left(\Pi^{*} A(b)\right) \cap C\right),
$$

where $C$ is the cartesian subgroup of the free product $\Pi^{*} A(b)$. We put for brevity, $\Lambda=V\left(\Pi^{*} A(b)\right) \cap C$, so that 


$$
K=\Pi^{*} A(b) / \Lambda ; \quad W=W^{*} / \Lambda
$$

Any element of $\Pi^{*} A(b)$ can be written uniquely in the normal form $a_{1}\left(b_{1}\right) \cdots a_{r}\left(b_{r}\right)$ where $a_{i} \in A, b_{i} \in B, i=1, \cdots, r ; b_{j} \neq b_{j+1}, j=1, \cdots$, $r-1$. Write

$$
\begin{aligned}
X & =\left\{a(1) a^{-1}(b) \mid a \in A, b \in B\right\} \\
& =\left\{\left[a^{-1}(1), b\right] \mid a \in A, b \in B\right\} \subset \Pi^{*} A(b) .
\end{aligned}
$$

Then the following is true.

4.1.4 LeMMA. If $a_{1}\left(b_{1}\right) \cdots a_{r}\left(b_{r}\right), a_{i} \in A, b_{i} \in B, b_{j} \neq b_{j+1}$, is any element of $\Pi^{*} A(b)$, then the element

lies in $\operatorname{sg} p(X)$.

$$
g=\left(a_{1} \cdots a_{r}\right)^{-1}\left(b_{1}\right) a_{1}\left(b_{1}\right) \cdots a_{r}\left(b_{r}\right)
$$

Proof. Any element of the form $a^{-1}\left(b^{\prime}\right) a\left(b^{\prime \prime}\right), a \in A ; b^{\prime}, b^{\prime \prime} \in B$, belongs to $\operatorname{sg} p(X)$ since

Write

$$
a^{-1}\left(b^{\prime}\right) a\left(b^{\prime \prime}\right)=\left(a^{-1}(1) a\left(b^{\prime}\right)\right)^{-1} a^{-1}(1) a\left(b^{\prime \prime}\right) \in \operatorname{sgp}(X) .
$$

Then

$$
\begin{aligned}
x_{1} & =a_{r}^{-1}(b)_{r} a_{r}\left(b_{r-1}\right), \\
x_{2} & =\left(a_{r-1} a_{r}\right)^{-1}\left(b_{r-1}\right)\left(a_{r-1} a_{r}\right)\left(b_{r-2}\right), \\
x_{r-1} & =\left(a_{2} a_{3} \cdots a_{r}\right)^{-1}\left(b_{2}\right)\left(a_{2} a_{3} \cdots a_{r}\right)\left(b_{1}\right) .
\end{aligned}
$$

$$
x_{1} x_{2} \cdots x_{r-1}=g^{-1},
$$

and since $x_{1} x_{2} \cdots x_{r-1} \in \operatorname{sg} p(X)$ by the preceding remark, the required result is obtained.

4.1.5 Corollary. In $W^{*},\left[B, \Pi^{*} A(b)\right]=\operatorname{sg} p(X)$.

Proof. Obviously

$$
\left[B, \Pi^{*} A(b)\right] \geqq \operatorname{sg} p(X) .
$$

To prove the reverse inclusion, we show that every generator $\left[b^{\prime}, k\right]$ of $\left[B, \Pi^{*} A(b)\right], b^{\prime} \in B, k \in \Pi^{*} A(b)$, lies in $\operatorname{sg} p(X)$. Suppose $k=a_{1}\left(b_{1}\right) \cdots a_{r}\left(b_{r}\right)$ is in the normal form. Then

$$
\begin{aligned}
{\left[b^{\prime}, k\right] } & =\left(a_{1}\left(b_{1} b^{\prime}\right) \cdots a_{r}\left(b_{r} b^{\prime}\right)\right)^{-1} a_{1}\left(b_{1}\right) \cdots a_{r}\left(b_{r}\right) \\
& =a_{r}^{-1}\left(b_{r} b^{\prime}\right) \cdots a_{1}^{-1}\left(b_{1} b^{\prime}\right) a_{1}\left(b_{1}\right) \cdots a_{r}\left(b_{r}\right) .
\end{aligned}
$$

Thus, by Lemma 4.1.4, $\left(a_{r}^{-1} \cdots a_{1}^{-1} a_{1} \cdots a_{r}\right)\left(b_{r} b^{\prime}\right)\left[b^{\prime}, k\right] \in \operatorname{sg} p(X)$; that is and the proof is complete.

$$
\left[b^{\prime}, k\right] \in \operatorname{sg} p(X)
$$


The next lemma is concerned with the free product $\Pi^{*} A(b)$ only.

4.1.6 Lemma. The epimorphism $\theta: \Pi^{*} A(b) \rightarrow A$ defined by $a(b) \theta=a$, $a \in A, b \in B$, (that is, $\theta$ amalgamates the $A(b))$ maps $A=V\left(\Pi^{*} A(b)\right) \cap C$ onto $(V \cap D)(A)$.

This is slightly surprising as one might expect on the face of it that $\Lambda \theta=V(A) \cap D(A)$ which, as remarked above, it not always the same as $(V \cap D)(A)$.

Before proving this lemma we need the following result which for clarity we state and prove separately.

When we say that a word in $F$ "involves" the variable $x_{i}$, we shall mean that the word contains $x_{i}$ or $x_{i}^{-1}$ when written in reduced form. A commutator with entries from $\left\{x_{1}^{ \pm 1}, x_{2}^{ \pm 1}, \cdots\right\}$ that involves $x_{i}$, takes the value 1 when $x_{i}$ is replaced by 1 . (See Higman [6], p. 169.)

4.1.7 Lemma ${ }^{2}$. The verbal subgroup $V \cap D$ is generated by the set of all those words $w=w\left(x_{1}, \cdots, x_{l}\right)$ in $V \cap D$ each of which can be written as a product $c_{1} \cdots c_{s}$ say, of commutators of weight $>1$ with entries from $\left\{x_{\mathbf{1}}^{ \pm \mathbf{1}}, \cdots, x_{l}^{ \pm \mathbf{1}}\right\}$, such that there exist two distinct subscripts $j$ and $k$ with the property that each commutator $c_{i}, 1 \leqq i \leqq s$, involves both $x_{j}$ and $x_{k}$.

PROOF. The proof is by induction on the number of variables a word in $V \cap D$ involves. If this number is 2 , the word already has the required form. Suppose that words in $V \cap D$ involving fewer than $l$ variables are products of words of the form $w$, and let $v=v\left(x_{1}, \cdots, x_{l}\right)$ be any element of $V \cap D$ which involves all of $x_{1}, \cdots, x_{l}$. Since $v \in D$, it can be written as a product of commutators of weight $>1$ in $x_{1}^{ \pm 1}, \cdots, x_{l}^{ \pm 1}$. By using the identity $y_{1} y_{2}=y_{2} y_{1}\left[y_{1}, y_{2}\right]$ repeatedly, we see that we can write

$$
v=v_{1} v_{2} v_{3} v_{4},
$$

where the $v_{i}, i=1,2,3,4$, are products of commutators of weight $>1$ in the $x_{i}$ 's and their inverses, and in $v_{1}$ all factors involve neither $x_{1}$ nor $x_{2}$; in $v_{2}$ all involve $x_{1}$ but not $x_{2}$; in $v_{3}$ all involve $x_{2}$ but not $x_{1}$; and the factors of $v_{4}$ all involve both $x_{1}$ and $x_{2}$. If we put successively $x_{1}=x_{2}=1 ; x_{1}=1$; $x_{2}=1$, in $v$, we see that $v_{1}, v_{2}, v_{3}, v_{4} \in V \cap D$. Now $v_{1}, v_{2}$ and $v_{3}$ involve at most $l-1$ variables and so, by the inductive hypothesis, they are products of words in $V \cap D$ of the right form. The element $v_{4}$ is already of the required form. Obviously no generality has been lost by working with the particular $l$ variables $x_{1}, \cdots, x_{l}$, and the proof is complete.

Proof of 4.1.6. Let $w=w\left(x_{1}, \cdots, x_{l}\right)$ be as in the statement of Lemma 4.1.7: $w=c_{1} \cdots c_{s}$ and the $c_{i}$ simultaneously involve the variables

2 This lemma was suggested by Professor Hanna Neumann as a correction of my original proof of 4.1.6. 
$x_{j}, x_{k}$. Since $B \neq E$, there exist $b^{\prime}, b^{\prime \prime} \in B$ for which $b^{\prime} \neq b^{\prime \prime}$. Substitute in $w$ any $l$ elements $g_{1}, \cdots, g_{l} \in A$, for $x_{1}, \cdots, x_{l}$ respectively. The resulting element is in $(V \cap D)(A)$. On the other hand, if we substitute $g_{i}\left(b^{\prime}\right)$ for $x_{i}, i \neq k$, and $g_{k}\left(b^{\prime \prime}\right)$ for $x_{k}$, the form of $w$ ensures that the resulting element is in $A$. Now

$$
w\left(g_{1}\left(b^{\prime}\right), \cdots, g_{k}\left(b^{\prime \prime}\right), \cdots, g_{\imath}\left(b^{\prime}\right)\right) \theta=w\left(g_{1}, \cdots, g_{\imath}\right),
$$

and since, by 4.1 .7 , all such elements $w\left(g_{1}, \cdots, g_{l}\right)$ generate $(V \cap D)(A)$, we have proved that

$$
\Lambda \theta \geqq(V \cap D)(A) .
$$

We now prove the reverse inclusion.

Let $R$ be the kernel of an epimorphism $\psi$ from a free group $F_{1}$ of suitable rank, onto $A: A \cong F_{1} / R$. Take $|B|$ isomorphic copies of $F_{1}$, denoted by $F_{1}(b), b \in B$. Then corresponding to $\psi$ we have, for each $b \in B$, the obvious epimorphism $\psi(b): F_{1}(b) \rightarrow A(b)$. Consider the free product $\prod_{b \in B}^{*} F_{1}(b)=\Pi^{*} F_{1}(b)$, say. Let $\phi$ be the epimorphism $\Pi^{*} F_{1}(b) \rightarrow \Pi^{*} A(b)$, whose restriction to $F_{1}(b)$ is $\psi(b)$. It then suffices to prove that
4.1.8
$(V \cap D)\left(\Pi^{*} F_{1}(b)\right) \phi \geqq \Lambda$.

For,

$$
(V \cap D)\left(\Pi^{*} F_{1}(b)\right) \phi \theta=(V \cap D)(A),
$$

and so, from 4.1.8,

$$
(V \cap D)(A) \geqq A \theta \text {. }
$$

4.1.8 is proved as follows. Let $v(x)=v\left(x_{1}, \cdots, x_{l}\right) \in V$ be such that for some $k_{1}, \cdots, k_{\imath} \in \Pi^{*} A(b)$, we have

$$
v\left(k_{1}, \cdots, k_{l}\right) \in C \text {. }
$$

Every element $g$ of $A=V\left(\Pi^{*} A(b)\right) \cap C$ is obtainable in this way from some $v(\boldsymbol{x}) \in V$. Suppose $f_{i} \phi=k_{i}$, where $f_{i} \in \Pi^{*} F_{1}(b), i=1, \cdots, l$. We now consider the element $v(f)=v\left(f_{1}, \cdots, f_{l}\right)$. Modulo its cartesian, $\Pi^{*} F_{1}(b)$ is the direct product of the $F_{1}(b)$. From this, together with the fact that the complete inverse image of $C$ under $\phi$ is the product of the cartesian of $\Pi^{*} F_{1}(b)$ and the normal closure in $\Pi^{*} F_{1}(b)$ of all $R(b)$, we see that we may write

$$
v(f)=d \cdot h_{1}\left(b_{1}\right) \cdots h_{t}\left(b_{t}\right),
$$

where $d$ belongs to the cartesian of $\Pi^{*} F_{1}(b) ; h_{i} \in R, b_{i} \in B, i=1, \cdots, t$, and $b_{j} \neq b_{k}$ for $j \neq k$.

Next suppose $\left\{y_{\gamma} \mid \gamma \in \Gamma\right\}$ ( $\Gamma$ some index set) is a set of free generators of $F_{1}$. Then $\left\{y_{\gamma}(b) \mid \gamma \in \Gamma, b \in B\right\}$ generates $\Pi^{*} F_{1}(b)$ freely and the $f_{i}$ may be regarded as reduced words in these generators. Consider the subset 
$S=\left\{y_{\gamma}(b) \mid y_{\gamma}(b)\right.$ occurs in $f_{i}$ for some $\left.i=1, \cdots, l\right\}$, and let $\mu: g p(S) \rightarrow F$ be the monomorphism extending any $(1,1)$ mapping from $S$ into the free generators $\left\{x_{1}, x_{2}, \cdots\right\}$ of $F$. Then $v(f) \mu \in V$. If, for some fixed $j, 1 \leqq j \leqq t$, we set $x_{i}=1$ in the word $v(f)$ whenever $x_{i} \mu^{-1} \notin F_{1}\left(b_{j}\right)$, then we find that also

$$
h_{j}\left(b_{j}\right) \mu \in V, \quad j=1, \cdots, t .
$$

Hence $d \mu \in V$; obviously $d \mu \in D$. Write $d \mu=v^{\prime}$.

Thus, for each element $g \in \Lambda$, we have found a word $v^{\prime} \in V \cap D$ such that the substitution $\mu^{-1} \phi$ gives $g: v^{\prime} \mu^{-1} \phi=g$. Now $v^{\prime} \mu^{-1} \in(V \cap D)$ $\left(\Pi^{*} F_{1}(b)\right)$ and hence $(V \cap D)\left(\Pi^{*} F_{1}(b)\right) \phi \geqq \Lambda$ as required. This completes the proof of 4.1.8 and thence of Lemma 4.1.6.

4.1.9 Corollary. For all $b \in B,(V \cap D)(A(b))$ is contained in $\operatorname{sg} p(X) \Lambda$.

Proof. Corollary 4.1.5 implies that $\operatorname{sg} p(X)$ is normal in $W^{*}$. Let $g$ be any element in $(V \cap D)(A)$. Then by Lemma 4.1.6 there exists an element $h=a_{1}\left(b_{1}\right) \cdots a_{r}\left(b_{r}\right)$ in $\Lambda$ such that $a_{1} \cdots a_{r}=g$. By Lemma 4.1.4 $g^{-1}\left(b_{1}\right) h \in \operatorname{sg} p(X)$, whence $g\left(b_{1}\right) \in \operatorname{sg} p(X) \Lambda$. The normality of $s g p(X)$ in $W^{*}$ then gives the stated result.

Finally we return to $W$ for which we obtain the following corollary. cosets

4.1.10 CoRollary. $[B, K]=\operatorname{sg} p(X) \Lambda / \Lambda=M$, where $M$ is the set of

$$
\begin{gathered}
\left.\left\{a_{1}\left(b_{1}\right) \cdots a_{r}\right) b_{r}\right) \Lambda \mid r \geqq 1 ; b_{1} \in B, a_{i} \in A, i=1, \cdots, r ; \\
\left.b_{j} \neq b_{j+1}, j=1, \cdots, r-1 ; a_{1} \cdots a_{r} \in(V \cap D)(A)\right\} .
\end{gathered}
$$

Proof. It is easy to see from 4.1.6 that the set $M$ forms a subgroup of $W$. Thus, since $X \Lambda / \Lambda \subset M$, we have $\operatorname{sg} p(X) \Lambda / \Lambda \leqq M$. To prove the reverse inclusion write $h=a_{1}\left(b_{1}\right) \cdots a_{r}\left(b_{r}\right)$, where $a_{1} \cdots a_{r} \in(V \cap D)(A)$. Then, by 4.1.4,

Therefore

$$
\left(a_{1} \cdots a_{r}\right)^{-1}\left(b_{1}\right) a_{1}\left(b_{1}\right) \cdots a_{r}\left(b_{r}\right) \in \operatorname{sg} p(X) .
$$

$$
h \in(V \cap D)\left(A\left(b_{1}\right)\right), \operatorname{sg} p(X)
$$

which by 4.1 .9 is contained in $\operatorname{sg} p(X) \cdot \Lambda$.

Proof of Theorem 4.1. The proof is immediate from Lemmas 4.1.1, 4.1.2 and Corollary 4.1.10).

For the application of Theorem 4.1 in $\S 5$, we require it only in the case $V(A)=E$. In this case, for convenience, we restate it as a corollary.

4.2 COROLlary. With the same notation as in Theorem 4.1, suppose further that $V(A)=E$. Then the normal closure of the normal subgroup $B_{1}>E$ of $B$ (with a transversal $T_{1}$ in $B$ ) in $W=A$ wor $_{3} B$, is $B_{1} M_{1}$ where 


$$
\begin{aligned}
M_{1} & =\left\{\alpha_{1}^{b_{1}} \cdots \alpha_{r}^{b_{r}} \mid r \geqq 1 ; b_{i} \in B_{1}, \alpha_{i} \in \prod_{t \in T_{1}}^{\mathfrak{g}} A(t),\right. \\
i & \left.=1, \cdots, r ; b_{j} \neq b_{j+1}, j=1, \cdots, r-1 ; \alpha_{1} \alpha_{2} \cdots \alpha_{r}=1\right\} .
\end{aligned}
$$

\section{Lower bound for $l\left(\mathfrak{R A}_{n}\right)$}

In this section we aim at proving that

$5.1 l\left(\mathfrak{R Q}_{n}\right) \geqq c$ for all $c \geqq 1$.

This, together with 2.2 , yields $l\left(\mathfrak{N A}_{n}\right)=c$ for $c>1$. (For $c=1, l\left(\mathfrak{R A}_{n}\right)=2$.)

For any variety $\mathfrak{B}$ we shall denote by $F_{k}(\mathfrak{B})$ the reduced free group of rank $k$ of $\mathfrak{B}$. As mentioned in $\S 1$, the method of proving 5.1 uses certain properties of the verbal wreath product

$$
W_{c}=F_{c}(\Re) w r_{\mathfrak{R}} C_{n}^{c},
$$

where $C_{n}^{c}$ is the direct product of $c$ isomorphic copies of $C_{n}$. Clearly $W_{c} \in \mathfrak{N A}_{n}$. We show that if $W_{c} \in \operatorname{var}\left(F_{c-1}\left(\mathfrak{R A}_{n}\right)\right)$ then $W_{c}$ is not only a factor of, but can be embedded in, a (finite) direct power of $F_{c-1}\left(\mathfrak{M Q R}_{n}\right)$. Hence there must be a set of normal subgroups of $W_{c}$, with trivial intersection, giving rise to factor groups embeddable in $\left.F_{c-1}(\mathfrak{N A})_{n}\right)$. We prove that, for all possible such sets, the factor group of at least one normal subgroup is not so embeddable.

In addition to Corollary 4.2, a few lemmas are needed. The following lemma has also been proved by A. L. Smel'kin [14]. His proof relies on the main theorem of that paper. We give a short, more direct proof.

5.2 Lemma. Let $\mathfrak{U}$ and $\mathfrak{B}$ be locally finite varieties of coprime exponents $m$ and $n$ respectively. Then the verbal wreath product

$$
W(k)=F_{k}(\mathfrak{U}) \text { wer } \mathfrak{u} F_{k}(\mathfrak{B})
$$

can be embedded in $F_{2 k}(\mathfrak{H B})$, the free group of rank $2 k$ of $\mathfrak{H B}$, for all $k \geqq 1$.

PRoof. Let $F_{2 k}$ be absolutely free on free generators $x_{1}, \cdots, x_{2 k}$. Then, by the Schur-Zassenhaus Theorem and the conditions of the lemma, $F_{2 k}(\mathfrak{U P B})=F_{2 k} / U\left(V\left(F_{2 k}\right)\right)$ is a splitting extension of $V\left(F_{2 k}\right) / U\left(V\left(F_{2 k}\right)\right)$ by $F_{2 k}(\mathfrak{B})=F_{2 k} / V\left(F_{2 k}\right)$.

If we write $F_{k}=\operatorname{sg} p\left\{x_{1}, \cdots, x_{k}\right\}$, the same remark applied to $F_{k}<F_{2 k}$ shows that there exists a set $T \subset F_{l k}$ which is a transversal for $V\left(F_{k}\right)$ in $F_{k}$ and is also, modulo $U\left(V\left(F_{2 k}\right)\right)$, a complement of $V\left(F_{k}\right)$ in $F_{k}$. Let $T_{1}$ be a right Schreier transversal for $V\left(F_{k}\right)$ in $F_{k}$. Since $V\left(F_{k}\right)=F_{k} \cap V\left(F_{2 k}\right)$, $T$ and $T_{1}$ are subsets of some transversals for $V\left(F_{2 k}\right)$ in $F_{2 k}$. For each $t_{1} \in T_{1}$ there exists a unique $t \in T$ such that 


\subsection{1 $t_{1}=a_{t} t$,}

where $a_{t}$ depends on $t$ and $a_{t} \in V\left(F_{k}\right)$. The mapping $T_{1} \rightarrow T$ so defined is $(1,1)$ and onto.

We now show briefly that one can choose a right Schreier transversal $T_{2}$ for $V\left(F_{2 k}\right)$ in $F_{2 k}$ which contains the set

$$
S=\left\{t_{1} x_{i}^{\alpha_{i}} \mid t_{1} \in T_{1}, 0 \leqq \alpha_{i} \leqq n-1, i=k+1, \cdots, 2 k\right\},
$$

by the following simple modification of the usual argument. It can easily be seen that the elements of $S$ lie in distinct cosets and have the Schreier property. Choose the elements of $S$ as representatives of their cosets. Let $Q$ be the set of cosets not so represented. To find suitable representatives for the cosets in $Q$ we use induction on the smallest length of elements in each coset in $Q$ (as in [8], vol. II, p. 33). Thus we obtain the required $T_{2}$. (See also M. J. Dunwoody [3].)

A set of free generators of $V\left(F_{2 k}\right)$ is then

$$
S_{2 k}=\left\{t_{2} x_{i}\left(\phi\left(t_{2} x_{i}\right)\right)^{-1} \mid i=1, \cdots, 2 k ; t_{2} \in T_{2}\right\} \backslash\{1\},
$$

where $\phi(g), g \in F_{2 k}$, is the element of $T_{2}$ representing the coset $g V\left(F_{2 k}\right)$. The set

$$
S_{k}=\left\{t_{1} x_{i}\left(\phi\left(t_{1} x_{i}\right)\right)^{-1} \mid i=1, \cdots, k, t_{1} \in T_{1}\right\} \backslash\{1\}
$$

is a set of free generators of $V\left(F_{k}\right)$. By the way $T_{2}$ was chosen, $S_{k} \subset S_{2 k}$. Write

$$
\begin{aligned}
X & =\left\{t_{2} x_{i}\left(\phi\left(t_{2} x_{i}\right)\right)^{-1} \mid t_{2}=t_{1} x_{i}^{n-1}, t_{1} \in T_{1}, i=k+1, \cdots, 2 k\right\} \\
& =\left\{\left(x_{i}^{n}\right)^{t_{1}^{-1}} \mid t_{1} \in T_{1}, i=k+1, \cdots, 2 k\right\} \subset S_{2 k} \backslash S_{k} .
\end{aligned}
$$

By 5.2.1,

Write

$$
\left(x_{i}^{n}\right)^{t_{1}^{-1}}=\left(\left(x_{i}^{n}\right)^{t^{-1}}\right)^{a_{t}^{-1}}, a_{t}^{-1} \in \operatorname{sg} p\left(S_{k}\right) .
$$

$$
Y=\left\{\left(x_{i}^{n}\right)^{t^{-1}} \mid t \in T, i=k+1, \cdots, 2 k\right\} .
$$

Since $a_{t} \in \operatorname{sg} p\left(S_{k}\right)$ for all $t \in T$ and $Y$ is obtained from $X \subset S_{2 k} \backslash S_{k}$ by suitable conjugation by the $a_{t}$, it follows that the set $\left(S_{2 k} \backslash X\right) \cup Y$ is an alternative set of free generators of $V\left(F_{2 k}\right)$.

Modulo $U\left(V\left(F_{2 k}\right)\right), \operatorname{sg} p\left\{x_{i}^{n} \mid i=k+1, \cdots, 2 k\right\}$ is isomorphic to $F_{k}(U)$. Because of the way $T$ was chosen, we have, modulo $U\left(V\left(F_{2 k}\right)\right)$,

$$
\begin{aligned}
& \operatorname{sg} p\left\{T, x_{i}^{n} \mid i=k+1, \cdots, 2 k\right\} \\
\cong & \operatorname{sg} p\left\{x_{i}^{n} \mid i=k+1, \cdots, 2 k\right\} w r_{\mathfrak{u}} \operatorname{sg} p(T)
\end{aligned}
$$

which is isomorphic $\left(\bmod U\left(V\left(F_{2 k}\right)\right)\right)$ to $W(k)$. This completes the proof.

5.3 Corollary. If $F_{\nu}(\mathfrak{W} \mathfrak{i})$ generates $\mathfrak{W} \mathfrak{H}$ for some cardinal $\nu$, then $W(k)$ can be embedded in some finite direct power of $F_{\nu}(\mathfrak{u} \mathfrak{B})$. 
Proof. Any free group of $\mathfrak{H B}$ can be embedded in some suitably large finite direct power of $F_{\nu}(\mathfrak{H} \mathfrak{B})$. In particular, this applies to $F_{2 k}(\mathfrak{U M B})$ and thus to $W(k)$ by Lemma 5.2 .

5.4 Lemma. If $n=p$, a prime, then every non-empty set of normal subgroups of $W_{c}=F_{c}(\Re) w r_{\mathfrak{R}} C_{n}^{c}$, such that none of the normal subgroups is wholly contained in the base group, has non-trivial intersection.

For the proof a corollary of the following lemma is needed.

5.4.1 Lemma. (Cf. Higman [6].) Let $F_{k}$ be (absolutely) free on $x_{1}, \cdots, x_{k}, k \geqq 2$, and let $\mathfrak{B}$ be a nilpotent variety of class $c$. Denote the subsets of $\left\{x_{1}, \cdots, x_{k}\right\}$ containing not less than 2 and not more than $c$ elements by $S_{1}, S_{2}, \cdots, S_{l}$, and form $C\left(S_{i}\right)=s g p\{\gamma \mid \gamma$ a commutator of weight $c$ with set of entries precisely $\left.S_{i}\right\}$. Then, if $\theta$ is the natural homomorphism $F_{k} \rightarrow F_{k} / V\left(F_{k}\right)$, we have

$$
\operatorname{sg} p\left\{C\left(S_{i}\right) \mid i=1, \cdots, l\right\} \theta=C\left(S_{1}\right) \theta \times \cdots \times C\left(S_{l}\right) \theta .
$$

Proof. It suffices to prove that if

$$
\gamma_{1} \gamma_{2} \cdots \gamma_{l} \in V\left(F_{k}\right)
$$

where $\gamma_{i} \in C\left(S_{i}\right), i=1, \cdots, l$, then

$$
\gamma_{i} \in V\left(F_{k}\right) \text { for each } i \text {. }
$$

We use induction on the cardinal of $S_{i}$. Let $\phi_{i}$ be the endomorphism of $F_{k}$ which fixes the elements of $S_{i}$ and maps all other free generators in $\left\{x_{1}, \cdots, x_{k}\right\}$ onto 1 . Then if $\left|S_{i}\right|=2$,

$$
\left(\gamma_{1} \gamma_{2} \cdots \gamma_{l}\right) \phi_{i}=\gamma_{1} \phi_{i} \gamma_{2} \phi_{i} \cdots \gamma_{l} \phi_{i}=\gamma_{i} .
$$

Therefore $\gamma_{i} \in V\left(F_{k}\right)$. Assume as inductive hypothesis that $\gamma_{j} \in V\left(F_{k}\right)$ for all $S_{j}$ with $\left|S_{j}\right| \leqq s<k$. We may then omit from $\gamma_{1} \cdots \gamma_{l}$ those $\gamma_{j}$ 's whose corresponding $S_{j}$ 's contain $\leqq s$ elements, and then have the remaining product still belonging to $V\left(F_{k}\right)$. If $\left|S_{i}\right|=s+1$, an application of $\phi_{i}$ to this smaller product completes the proof of the inductive step and thence the proof of the lemma.

We apply this lemma to the base group $K$ of $W_{c}$. Suppose $x_{1}, \cdots, x_{\theta}$ freely generate $F_{c}(\mathfrak{N})$, the bottom group of $W_{c}$. Then there is a commutator of weight $c$ with its set of entries precisely $\left\{x_{1}, \cdots, x_{c}\right\}$ which does not reduce to 1 . Otherwise, since $F_{c}(\mathfrak{N})$ is reduced free, all commutators of weight $c$ would be 1 and $F_{0}(\Re)$ would be nilpotent of class $<c$. Denote such a commutator by $\gamma\left(x_{1}, \cdots, x_{c}\right)$. An obvious set of free generators of $K$ is

Then we have

$$
\left\{x_{i}(b) \mid i=1, \cdots, c ; b \in C_{n}^{o}\right\}
$$


5.4.2 CoRollary. The elements of the set

$$
\left\{\gamma\left(x_{1}\left(b_{1}\right), \cdots, x_{c}\left(b_{c}\right)\right) \mid b_{i} \in C_{n}^{c}\right\},
$$

are all non-trivial of order $m_{1}$ where $m_{1}$ divides $m$, the exponent of $\mathfrak{R}$, and independent. If $m_{1}=q m_{2}$ where $q$ is a prime, then

$$
S=\left\{\left(\gamma\left(x_{1}\left(b_{1}\right), \cdots, x_{c}\left(b_{c}\right)\right)\right)^{m_{2}} \mid b_{i} \in C_{n}^{c}\right\}
$$

is a basis for a direct product of $n^{c^{2}}$ isomorphic copies of $C_{q}$, considered as a vector space of dimension $n^{c^{2}}$ over $G F(q)$.

Proof of Lemma 5.4. We shall use P. Hall's well known theorem (see $[5], p .141)$ the relevant parts of which we shall state here for convenience.

5.4.3 Let $G$ be a soluble group of order $r$ s where $(r, s)=1$. Then

5.4.4 $G$ has at least one subgroup of order $r$;

5.4.5 any two subgroups of order $r$ are conjugate;

5.4.6 any subgroup whose order divides $r$, is contained in a subgroup of order $r$.

Since $\mathfrak{R A}_{n}$ is a soluble, locally finite variety, we may apply this theorem to any of its finitely generated groups.

We have $\left|W_{a}\right|=p^{c} t$ where $(p, t)=1$. (We are now dealing with the case $W_{c}=F_{c}(\mathfrak{N}) w r_{\mathfrak{R}} C_{p}^{c}$ where $p$ is a prime.) The base group $K$ has order $t$ and $K \triangleleft W_{\mathrm{c}}$. By 5.4.5. $K$ is then the only subgroup of order $t$ and by 5.4.6 $K$ contains every subgroup of order prime to $p$. Let $N$ be any normal subgroup of $W_{c}$ not wholly contained in $K$. Suppose $|N|=p^{\alpha} t_{1}$ where $\left(p, t_{1}\right)=1$. Then by the above, $\alpha>0$. By 5.4.4 $N$ contains a subgroup of order $t_{1}$ and this must be $N \cap K$. Again by 5.4.4 $N \cap K$ is complemented in $N$ by $B$, say, and by 5.4.5, 5.4.6 we may choose $B$ to be a subgroup of the top group $C_{p}^{c}$ of $W_{c}$.

Thus the truth of the lemma will follow if we show that the intersection of the normal closures in $W_{c}$ of all $p$-cycles contained in $C_{p}^{c}$, is non-trivial. This we proceed to do.

Denote the elements of $S$ (5.4.2) by $\gamma_{1}, \cdots, \gamma_{s}$ where $s=p^{c^{2}}$. We shall prove that an element of $K$ of the form

$$
x=\gamma_{1}^{l_{1}} \cdots \gamma_{s}^{l_{s}}
$$

lies in the above-mentioned intersection for some $l_{1}, \cdots, l_{s}$ not all $\equiv 0$ $(\bmod q)$.

Let $\Gamma \cong C_{p}$ be any $p$-cycle in the top group $C_{p}^{c}$ of $W_{c}$ and let $T$ be any transversal for $\Gamma$ in $C_{p}^{c}$. Then $|T|=p^{c-1}$. Denote by $\phi_{\Gamma}$ the mapping of 
$C_{p}^{o}$ into itself which sends each element onto its representative in $T$. Then let $\phi_{\Gamma}^{\prime}: s g p(S) \rightarrow s g p(S)$ be the homomorphism obtained by extending the mapping defined by

$$
\gamma\left(x_{1}\left(b_{1}\right), \cdots, x_{c}\left(b_{c}\right)\right) \phi_{\Gamma}^{\prime}=\gamma\left(x_{1}\left(b_{1} \phi_{\Gamma}\right), \cdots, x_{c}\left(b_{c} \phi_{\Gamma}\right)\right) .
$$

By Corollary $4.2, \gamma_{1}^{l_{1}} \cdots \gamma_{s}^{l_{*}} \in \Gamma^{W_{c}}$ if and only if

$$
\left(\gamma_{1}^{l_{1}} \cdots \gamma_{s}^{l_{s}}\right) \phi_{\Gamma}^{\prime}=1
$$

If we collect the $\gamma_{i}^{\prime}$ 's which have become identified under $\phi_{\Gamma}^{\prime}$, then in the collected expression we must have powers of distinct $\gamma_{i}$ 's congruent to $0(\bmod q)$ for 5.4.7 to be satisfied. In this way we are led to $|T|^{c}=p^{c(c-1)}$ linear homogeneous equations in $l_{1}, \cdots, l_{\mathrm{s}}$ over $G F(q)$. Thus $\gamma_{1}^{l_{1}} \cdots \gamma_{s}^{l_{s}} \in \Gamma^{W_{\mathrm{c}}}$ if and only if $l_{1}, \cdots, l_{s}$ is a solution of this system of equations. It follows that if $x$ is to lie in the intersection of the normal closures of all $p$-cycles contained in $C_{p}^{e}, l_{1}, \cdots, l_{s}$ must be a solution simultaneously of the corresponding systems of linear equations.

Since there are $\left(p^{c}-1\right) /(p-1)$ distinct $p$-cycles in $C_{p}^{c}$, we get in all $\left(p^{c}-1\right) /(p-1) \cdot p^{c(c-1)}$ (not necessarily independent) equations whose solutions are precisely the admissible values for $l_{1}, \cdots, l_{s}$. Now

$$
s=p^{c^{2}}>\frac{p^{c-1}}{p-1} \cdot p^{c(c-1)}
$$

for all $p$ and therefore there exist non-trivial solutions. This completes the proof.

Proof of $5.1: l\left(\mathfrak{N A}_{n}\right) \geqq c$. Suppose $F_{c-1}\left(\mathfrak{N A}_{n}\right)$ generates $\mathfrak{N A}_{n}$. Then, by Corollary 5.3, we can find a (finite) set $\Sigma$ of normal subgroups of $W_{c}$ with trivial intersection and with factor groups embeddable in $F_{c-1}\left(\mathfrak{R A}_{n}\right)$. Let $p$ be any prime dividing $n$, and consider the unique subgroup $H$ of the top group $C_{n}^{c}$ of $W_{c}$ such that $H \cong C_{p}^{c}$. At least one normal subgroup, say $M \in \Sigma$, must intersect $H$ trivially. For otherwise Lemma 5.4 together with Lemma 4.1.1 would tell us that $\bigcap_{M \in \Sigma} M \neq E$. Thus $W_{\mathrm{c}} / M$ contains a subgroup isomorphic to $C_{p}^{c}$ and therefore, by our supposition, so does $F_{c-1}\left(\mathfrak{R A}_{n}\right)$. If $F_{c-1}\left(\mathfrak{N A}_{n}\right)$ contains a subgroup $G \cong C_{p}^{\mathfrak{c}}$, then, by 5.4.3, $G$ is contained in a subgroup isomorphic to $C_{n}^{c-1}$, which is impossible. We have reached a contradiction and the proof is complete.

\section{References}

[1] Gilbert Baumslag, B. H. Neumann, Hanna Neumann and Peter M. Neumann, 'On varieties generated by a finitely generated group'. Math. Z. 86 (1964), 93-122.

[2] R. G. Burns, Ph. D. thesis. Australian National University, 1966. 
[3] M. J. Dunwoody, 'On verbal subgroups of free groups'. Arch. Math., 16 (1965), 153-157.

[4] Wolfgang Gaschütz, 'Uber die $\Phi$-Untergruppe endlicher Gruppen'. Math. $Z$., 58 (1953), $160-170$.

[5] Marshall Hall, Jr., The theory of groups. (New York 1959).

[6] Graham Higman. 'Some remarks on varieties of groups'. Quarterly J. Math., Oxford (2) 10 (1959), 165-178.

[7] L. G. Kovács and M. F. Newman. 'Cross varieties of groups'. Submitted to Proc. Roy. Soc.

[8] A. G. Kurosh. The theory of groups, Vols. 1 and 11. (New York 1956).

[9] S. Moran. Associative operations on groups I. Proc. London Math. Soc. (3) 6 (1956), $581-596$.

[10] S. Moran, 'The homomorphic image of the intersection of a verbal subgroup and the cartesian subgroup of a free product'. J. London. Math. Soc., 33 (1958), 237-245.

[11] B. H. Neumann. 'Identical relations in groups I'. Math. Ann., 114 (1937), 506-525.

[12] Peter M. Neumann. 'On the structure of standard wreath products of groups'. Math. $Z$., 84 (1964) $343-373$.

[13] Sheila Oates and M. B. Powell, 'Identical relations in finite groups'. J. Algebra, 1 (1964), $11-39$.

[14] A. L. Smel'kin. 'Wreath products and varieties of groups'. Izv. Nauk S.S.S.R. Ser. Mat., 29 (1965), 149-170. (Also for summary in English: Soviet Mathematics. Vol. 5. No. 4 (1964). Translation of Dokl. Akad. Nauk S.S.S.R. for Am. Math. Soc).

[15] Paul M. Weichsel, 'On critical p-groups'. Proc. London Math. Soc. 14 (1964), 83-100.

\section{Monash University}

Melbourne 